\title{
High Performance Computing and the Progress of Weather and Climate Forecasting
}

\author{
Philippe Bougeault \\ European Centre for Medium-Range Weather Forecasts, UK
}

\begin{abstract}
Over the last few years, global weather forecasts from a number of operational numerical weather prediction centres have continued to progress steadily in skill and reach an impressive level of quality. The drivers of progress are increased resolution, better numerical algorithms, massive amounts of new observations from satellites, and very advanced data assimilation schemes to ingest observations in models. All of these aspects rely heavily on the ever increasing computing resources that have become available. The first part of the talk will describe some aspects of these improvements and will give considerations on how much each type of improvement has actually contributed to the overall improvement in forecast skill.

While the most advanced weather forecasting models have reached global horizontal resolutions of about $20 \mathrm{~km}$, climate models are rather ranging from $300 \mathrm{~km}$ to $100 \mathrm{~km}$. Recent research points to the need to increase the resolution of climate models to benefit from the same large improvements as the weather forecast models have undergone. In fact the concept of seamless weather and climate forecasts is rapidly developing. The second part of the talk will focus on this concept.

The third part of the talk will highlight current difficulties in developing highly scalable systems, based on the ECMWF example. The ECMWF high performance computer system consists of 2 IBM POWER5+ Cluster 1600 supercomputer systems. Each cluster is made up of 155 compute nodes, plus a further 10 nodes which are used for I/O and networking. Each node consists of 8 Dual-core Power5+ processors, ie 16 physical cores, which have a clock frequency of $1.9 \mathrm{GHz}$., a peak performance of 7.6 Gflops/core and a sustained performance of 1 Gflop/core. Simultaneous Multi-Threading (SMT) means that each physical core can run 2 threads concurrently. So with SMT switched on, each node can be considered to have 32 logical cores. Users specify jobs by the number of MPI processes and the number of OpenMP threads. I will show examples of work to improve the scalability of various parts of our forecasting system on this machine.
\end{abstract}

